\title{
Revista Colombiana de

\section{Estudio EPRAS: estudio poblacional del riesgo cardiovascular de una población colombiana}

\author{
Camilo Alvarado ${ }^{\mathrm{a}, *}$, Dora I. Molina ${ }^{\mathrm{b}}$, Alejandro Zárate ${ }^{\mathrm{c}}$ y Esteban Toro ${ }^{\mathrm{c}}$ \\ a Departamento de Medicina Interna, Fundación Santa Fe, Bogotá, Colombia \\ b Departamento Clínico, Facultad de Ciencias para la Salud, Universidad de Caldas, Manizales, Colombia \\ c Semillero de Investigación en Medicina Interna MEDICAL, Universidad de Caldas, Manizales, Colombia
}

Recibido el 23 de julio de 2013; aceptado el 18 de junio de 2014

Disponible en Internet el 26 de noviembre de 2014

\author{
PALABRAS CLAVE \\ Hipertensión arterial; \\ Tabaquismo; \\ Enfermedad \\ cardiovascular; \\ Hipercolesterolemia; \\ Sedentarismo
}

\begin{abstract}
Resumen
Objetivos: Describir el comportamiento de los factores de riesgo relacionados con la hipertensión arterial, el consumo declarado de medicamentos y el perfil metabólico en una población de pacientes valorados en el Servicio de Medicina Interna del Hospital Departamental Felipe Suárez de Salamina, Caldas, durante octubre a diciembre de 2012.

Materiales y métodos: Se valoraron 400 pacientes hipertensos del servicio de Medicina Interna del Hospital Departamental Felipe Suárez, durante octubre a diciembre de 2012. Se aplicaron formatos de registro y se procesaron en forma descriptiva.

Resultados: Se encontró una prevalencia de sedentarismo del 43,7\% y de tabaquismo del 9,3\%. En un $84,3 \%$ de los hipertensos evaluados se encontraron cifras de presión arterial controladas. El 73,3\% tenía pobre control de las cifras de colesterol LDL. Los diuréticos fueron el grupo de medicamentos antihipertensivos de mayor consumo $(87,2 \%)$.

Conclusiones: La prevención y el control de los factores de riesgo cardiovascular deben convertirse en una prioridad, en una política pública saludable del sistema de salud, que impacte a la totalidad de la población, con el fin de disminuir la prevalencia ascendente de los denominados grandes síndromes del adulto, responsables de las mayores tasas relacionadas con morbimortalidad en la población general, a través de la promoción de estilos de vida saludables, realización de actividad física regular y detección temprana de factores de riesgo.

(c) 2013 Sociedad Colombiana de Cardiología y Cirugía Cardiovascular. Publicado por Elsevier España, S.L.U. Todos los derechos reservados.
\end{abstract}

\footnotetext{
* Autor para correspondencia.

Correo electrónico: camilo.alvarado5@gmail.com (C. Alvarado).
} 


\section{KEYWORDS}

Hypertension; Smoking;

Cardiovascular

disease;

Hypercholesterolemia;

Sedentarism
EPRAS Study: population-based study of cardiovascular risk in a Colombian population

\begin{abstract}
Objectives: To describe the behavior of risk factors associated with hypertension, reported consumption of drugs and metabolic profile in a population of patients evaluated in the internal medicine department of the Hospital Departamental Felipe Suárez of Salamina (in Caldas, Colombia) during the period between October and December 2012.

Materials and methods: We evaluated 400 patients with hypertension of the internal medicine department of the Hospital Departamental Felipe Suárez during the months of October, November and December 2012. Registration forms were applied and processed in descriptive form.

Results: We found a prevalence of sedentarism of $43.7 \%$ and smoking of $9.3 \%$. In $84.3 \%$ of hypertensive patients evaluated we found controlled blood pressure. $73.3 \%$ had poor control of LDL cholesterol levels. The diuretics were the group of anti hypertensive medication with the highest consumption (87.2\%).

Conclusions: primary prevention and control of cardiovascular risk factors should be turned into a priority, healthy public policy in the health system, which impacts the entire population, in order to decrease the rising prevalence of so-called large adult syndromes and those which are responsible for the higher rates associated with morbidity and mortality in the general population through promotion of healthy lifestyles, physical activity and early detection of risk factors.

(c) 2013 Sociedad Colombiana de Cardiología y Cirugía Cardiovascular. Published by Elsevier España, S.L.U. All rights reserved.
\end{abstract}

\section{Introducción}

El creciente interés académico relacionado con el riesgo cardiovascular, marca un poco la tendencia epidemiológica de las poblaciones actuales. En tal sentido, las enfermedades crónicas no transmisibles ocupan gran proporción de las patologías más prevalentes en nuestra comunidad y generan un porcentaje muy elevado de morbimortalidad en la población general. Se conoce que la enfermedad cardiovascular es la causa más importante de muerte en todo el mundo; tan significativo es el cambio epidemiológico, que algunos la llaman la pandemia del siglo XXI ${ }^{1}$. Parece profética la declaración del año 1969 sobre el riesgo cardiovascular, realizada por el comité ejecutivo de la Oganización Mundial de la Salud al señalar que: «la mayor epidemia de la humanidad, la enfermedad cardiovascular, alcanzará proporciones enormes y afectará cada vez más a personas más jóvenes. En los próximos años se convertirá en la mayor epidemia de la humanidad si no somos capaces de cambiar esta tendencia mediante la concentración de esfuerzos investigadores sobre su causa y prevención $»^{2}$. Del mensaje anterior radica la importancia de investigar el riesgo cardiovascular en poblaciones colombianas. Experiencias pasadas muestran cómo la extrapolación de resultados de investigaciones hechas bajo otros términos y en poblaciones que distan mucho de la nuestra, no generan el impacto suficiente en la disminución del riesgo cardiovascular.

La enfermedad aterosclerótica es un trastorno crónico, silente y de lenta progresión, que, en general, expresa síntomas cuando se halla en estadios avanzados; de allí la importancia de hacer seguimientos clínicos estrictos mediante programas donde se evalúen diferentes variables que permitan la identificación temprana y el tratamiento oportuno de pacientes con riesgo cardiovascular elevado.

Se han concentrado grandes recursos económicos y esfuerzos científicos para establecer los lineamientos sobre la detección temprana y el tratamiento de los pacientes con algún grado de riesgo cardiovascular. Reflejo de ello son las múltiples publicaciones científicas al respecto en los últimos 20 años $^{3}$. En concordancia, a partir de la publicación del «Séptimo comité para la prevención, detección, evaluación y tratamiento de la presión arterial», han surgido gran cantidad de recomendaciones respecto a la evaluación de la hipertensión arterial en prevención primaria y secundaria; las mismas permanecen vigentes y permiten una aproximación a la valoración adecuada del riesgo, así como a la realización de intervenciones terapéuticas tempranas para el control y la disminución del riesgo relativo ${ }^{4}$. Similar es el caso de las recomendaciones con relación al manejo de pacientes con dislipidemia, cuyo pilar fundamental para su manejo en intervención primaria y secundaria durante la última década, se conoce como tercer reporte del panel de expertos sobre detección, evaluación y tratamiento del colesterol sanguíneo en adultos (Adult treatment panel III, o ATP III), en el cual se resumen las principales recomendaciones para el control metabólico relacionado con los niveles de lípidos séricos ${ }^{5}$. Estos son solo algunos ejemplos de esfuerzos de magnitud mundial en la lucha por la disminución de la morbimortalidad asociada con eventos cardiovasculares.

En un estudio publicado en $2012^{6}$, respecto a factores de riesgo relacionados con el estilo de vida, hallazgos electrocardiográficos y medicación antihipertensiva de pacientes 
valorados por el Servicio de Cardiología de la Fundación Santa Fe de Bogotá se encontraron tasas de hipertensión arterial del $57,1 \%$, tabaquismo del $11,7 \%$ y sedentarismo del $75 \%$, en el contexto de estratos socioeconómicos altos de la capital. En esta misma publicación se determinó que el grupo de medicamentos antihipertensivos de mayor consumo en dicha población fueron los antagonistas del receptor de angiotensina ॥, con un 55\%.

El conocimiento sobre el comportamiento de los factores de riesgo cardiovascular relacionados con el estilo de vida en la población general, específicamente en estratos socioeconómicos colombianos bajos, se basa principalmente en encuestas y fichas de notificación. Esta información se genera a partir de los programas de riesgo cardiovascular en centros de salud y hospitales públicos de primer y segundo nivel $y$ es recolectada por los entes territoriales, que a su vez ejercen vigilancia y control sobre los mismos. Pese a ello, no hay suficiente evidencia científica ni estudios poblacionales relacionados con el comportamiento de los factores de riesgo cardiovascular en estos sectores poblacionales. Los factores que implican posibles diferencias relacionadas con el acceso a los servicios de salud del llamado régimen subsidiado, incluyen: prescripción de medicamentos genéricos, solicitud de exámenes complementarios, acceso a consulta médica especializada y a programas de rehabilitación cardiovascular, aspectos fundamentales relacionados con el control de la hipertensión arterial y con la reducción de la morbimortalidad asociada a las enfermedades cardiovasculares.

Se presenta un estudio poblacional de pacientes hipertensos que fueron valorados por el Servicio de Medicina Interna del Hospital Departamental Felipe Suárez, en quienes se determinó la prevalencia de factores de riesgo cardiovascular asociados, control metabólico y medicamentos antihipertensivos de la prescripción médica.

\section{Objetivo}

\section{General}

Describir el comportamiento de factores de riesgo relacionados con la hipertensión arterial, el consumo declarado de medicamentos y el perfil metabólico en una población de pacientes valorados en el Servicio de Medicina Interna del Hospital Departamental Felipe Suárez, de Salamina, Caldas, durante octubre a diciembre de 2012.

\section{Específicos}

1. Describir los aspectos sociodemográficos presentes en la población de usuarios valorada.

2. Determinar la presencia de factores de riesgo cardiovascular asociados con el estilo de vida, por medio de entrevista personal y corroboración a través de la historia clínica.

3. Identificar y describir los medicamentos consumidos por la población incluida en el estudio.

4. Describir las variables básicas relacionadas con el control metabólico en el grupo de usuarios incluidos en el estudio.

\section{Metodología}

\section{Población}

Se valoraron 400 usuarios hipertensos del régimen subsidiado del Servicio de Medicina Interna (consulta externa) del Hospital Departamental Felipe Suárez, de Salamina, Caldas, durante octubre a diciembre de 2012.

\section{Criterios de inclusión}

Pacientes mayores de 50 años, usuarios del régimen subsidiado, valorados por el Servicio de Medicina Interna en consulta externa, con diagnóstico de hipertensión arterial.

\section{Criterios de exclusión}

Pacientes menores de 50 años, discapacitados o comprometidos en su esfera mental, con afiliación al sistema de salud diferente al régimen subsidiado $\mathrm{y} \mathrm{y} / \mathrm{o}$ acudientes que manifestaron su desacuerdo con el consentimiento informado y la entrevista personal.

\section{Diseño}

Estudio poblacional descriptivo de corte transversal.

\section{Muestreo}

Se calculó un tamaño de muestra a partir de una población estimada de 18.345 habitantes con la fórmula $n: \mathrm{N \sigma}^{2} \mathrm{Z}^{2}$ / $(\mathrm{N}$ 1) $\mathrm{e}^{2}+\sigma^{2} Z^{2}$ ( $\sigma$ : desviación estándar estimada $0,5, Z$ : valor obtenido por nivel de confianza del $95 \%$ en 1,96, e: límite aceptable de error en la muestra de 5\%), adquiriendo un estimativo de 376 habitantes. Se valoraron 400 pacientes que cumplían los criterios de inclusión para mayor significación estadística.

\section{Fuentes de información}

Usuarios, familiares o acudientes de la Institución, historia clínica de consulta externa; resultados de laboratorio (colesterol total, colesterol LDL, colesterol HDL, triglicéridos, glucemia basal y creatinina) y la estadística realizada con base en los datos obtenidos.

\section{Procedimiento}

Se realizó una entrevista personal con usuarios del Servicio de Medicina Interna, en el área de consulta externa. Una vez se informó al paciente, se aplicó un formato de registro para información personal y factores de riesgo cardiovascular asociados con el estilo de vida. Se tomó la presión arterial, se registró y se indagó a los usuarios hipertensos acerca de su medicación actual; se corroboró en la historia clínica y se registró en el formato. Se describió y analizó el resultado de perfil lipídico, glucemia basal y creatinina en un formato diseñado para tal fin, que incluyó parámetros como: colesterol total, LDL, HDL; triglicéridos, glucemia basal y creatinina. 


\section{Consideraciones éticas}

La recolección de los datos se llevó a cabo bajo la tutoría de especialistas del área clínica vinculados al Hospital Departamental Felipe Suárez. Se efectuó una revisión de los resultados útiles de la historia clínica para la descripción estadística, sin obtener beneficios económicos. Se guardó la confidencialidad profesional y se mantuvo en reserva la identificación de los pacientes incluidos en el estudio.

\section{Variables}

Se observan en la tabla 1.

\section{Materiales y métodos}

Durante la recolección de los datos se utilizaron tres formatos de registro: uno descriptivo para factores de riesgo cardiovascular relacionados con el estilo de vida, uno para consignación de resultados de laboratorio y uno para consumo declarado de medicamentos.

\section{Procesamiento de la información}

La base de datos en SPSS 16.0 (licencia Universidad de Caldas) se digitó por duplicado y luego fue verificada por dos asesores de la investigación para eliminar errores en la transcripción de los datos. El análisis se llevó a cabo con estadísticos descriptivos: media, mediana, desviación estándar y valor mínimo y máximo para variables cuantitativas, y frecuencias absolutas y relativas para variables cualitativas.

\section{Resultados}

\section{Aspectos sociodemográficos}

Un total de 400 pacientes hipertensos valorados en el Servicio de Medicina Interna en la sección de consulta externa del Hospital Felipe Suárez, en el período de octubre a diciembre de 2012, cumplieron los criterios de inclusión. En las figuras 1 y 2 se observa la distribución de la población estudiada por sexo y edad. Un $35,3 \%$ de dicha población tenía diagnóstico confirmado de hipertensión arterial

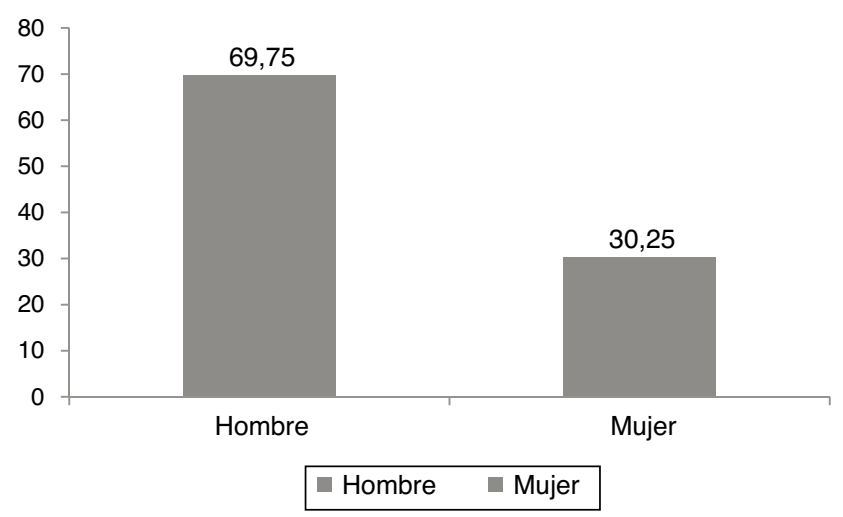

Figura 1 Distribución por sexo.

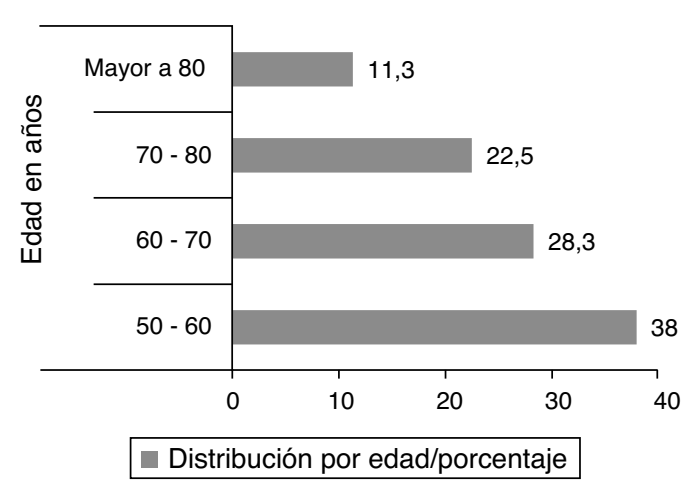

Figura 2 Distribución por edad.

10 años atrás, un 37,5\% había sido diagnosticado entre 5 y 10 años antes, un $18,8 \%$ entre 1 y 5 años y un 7,5\% hacía menos de un año.

\section{Factores de riesgo cardiovascular coexistentes}

Se evidenció coexistencia de diabetes mellitus tipo 2 en el $19,3 \%(n=77)$ de la población estudiada, con mayor proporción en el grupo de 61 a 70 años de edad $(23,89 \%)$, seguido por el grupo de mayores de 80 años, con prevalencia del $20 \%$. En la distribución por sexo, 23,29\% de las mujeres hipertensas padecía diabetes mellitus 2 y $9,9 \%$ de los hombres eran hipertensos. Respecto a la prevalencia de infarto agudo del miocardio, se reportó un $1,8 \%(n=7)$, más frecuente en mujeres y mayor proporción de casos en el grupo de 71 a 80 años (2,2\%). En el grupo de estudio se encontró una prevalencia de insuficiencia cardíaca congestiva del $1,8 \%(n=7)$, la cual fue más alta en hombres $(3,3 \%)$ y tuvo mayor distribución por rangos de edad entre los 61 y 70 años (2,65\%). La prevalencia de enfermedad cerebrovascular fue del $1 \%$ $(n=4)$ y la de enfermedad pulmonar obstructiva crónica del $3,5 \%(n=14)$; esta última afectó más a mujeres $(3,94 \%)$ y al rango de edad entre 71 y 80 años $(8,88 \%)$.

\section{Factores de riesgo relacionados con el estilo de vida y el control de las cifras de presión arterial}

Se encontró prevalencia de tabaquismo en el 9,3\% de la población estudiada, dividida en $8,96 \%$ para mujeres y $9,91 \%$ para hombres. De acuerdo con la edad, se evidenció mayor frecuencia de tabaquismo en el grupo de 50 a 60 años $(11,18 \%)$, seguido por el rango de edad entre $61 \mathrm{y}$ 70 años $(10,61 \%)$. Igualmente, se valoró la realización de actividad física y el sedentarismo en el grupo poblacional estudiado. Un 3,8\% de los pacientes hipertensos que eran sedentarios, declararon tabaquismo activo. El 56,25\% realizaba algún tipo de actividad física, la cual se clasificó en las siguientes categorías: camina menos de una hora al día, camina más de una hora al día, hace ejercicio menos de tres días a la semana y hace ejercicio más de tres días a la semana. La distribución de la población por actividad física realizada se observa en la figura 3 . En el $84,3 \%$ de los hipertensos evaluados se encontraron cifras de presión arterial controladas dentro de las metas de manejo, con distribución del 84,58 y del $83,47 \%$ para mujeres y hombres, respectivamente. La prevalencia de hipertensión arterial controlada 
Tabla 1 Variables epidemiológicas de la población estudiada

\begin{tabular}{|c|c|c|c|c|}
\hline \multicolumn{5}{|c|}{ Factores sociodemográficos, antecedentes cardiovasculares y medicamentos } \\
\hline \multirow{2}{*}{$\begin{array}{l}\text { Variable } \\
\text { independiente } \\
\text { 1. Edad }\end{array}$} & Descripción & \multirow{2}{*}{$\begin{array}{l}\text { Medición } \\
\text { Ordinal }\end{array}$} & \multicolumn{2}{|c|}{ Codificación } \\
\hline & Número de años actuales por rangos & & 1 & $=50-60$ años \\
\hline & & & & $=61-70$ años \\
\hline & & & & $=71-80$ años \\
\hline & & & 4 & $=$ mayor de 80 años \\
\hline \multirow[t]{2}{*}{ 2. Sexo } & & Nominal & 1 & $=$ mujer \\
\hline & & & 2 & $=$ hombre \\
\hline \multirow[t]{4}{*}{ 3. Años de HTA } & $\begin{array}{l}\text { Duración en años desde el diagnóstico } \\
\text { de hipertensión arterial }\end{array}$ & Ordinal & 1 & $=$ menos de 1 año \\
\hline & & & 2 & $=$ entre 1 y 5 años \\
\hline & & & 3 & $=$ entre 5 y 10 años \\
\hline & & & 4 & $=$ más de 10 años \\
\hline \multirow[t]{2}{*}{$\begin{array}{l}\text { 4. Infarto agudo } \\
\text { de miocardio }\end{array}$} & $\begin{array}{l}\text { Antecedente de infarto agudo de } \\
\text { miocardio }\end{array}$ & Nominal & 1 & $=$ presente \\
\hline & & & 2 & $=$ ausente \\
\hline \multirow[t]{2}{*}{$\begin{array}{l}\text { 5. Insuficiencia } \\
\text { cardíaca }\end{array}$} & $\begin{array}{l}\text { Antecedente de insuficiencia cardíaca } \\
\text { congestiva }\end{array}$ & Nominal & 1 & $=$ presente \\
\hline & & & 2 & $=$ ausente \\
\hline \multirow{2}{*}{$\begin{array}{l}\text { 8. Diabetes } \\
\text { mellitus }\end{array}$} & Antecedente de diabetes mellitus & Nominal & 1 & $=$ presente \\
\hline & & & 2 & $=$ ausente \\
\hline \multirow[t]{2}{*}{$\begin{array}{l}\text { 9. Enfermedad } \\
\text { cerebrovascular }\end{array}$} & $\begin{array}{l}\text { Antecedente de enfermedad } \\
\text { cerebrovascular (excluyendo accidente } \\
\text { isquémico transitorio) }\end{array}$ & Nominal & 1 & $=$ presente \\
\hline & & & 2 & $=$ ausente \\
\hline \multirow[t]{2}{*}{ 11. Tabaquismo } & $\begin{array}{l}\text { Consumo actual de cigarrillos (10 a } 20 \\
\text { cigarrillos/día) }\end{array}$ & Nominal & 1 & $=$ presente \\
\hline & & & 2 & $=$ ausente \\
\hline \multirow[t]{2}{*}{$\begin{array}{l}\text { 13. Actividad } \\
\text { física }\end{array}$} & $\begin{array}{l}\text { Realización de algún tipo de actividad } \\
\text { física diferente a la cotidiana }\end{array}$ & Nominal & 1 & $=$ presente \\
\hline & & & 2 & $=$ ausente \\
\hline \multirow[t]{5}{*}{$\begin{array}{l}\text { 14. Tipo de } \\
\text { actividad física }\end{array}$} & $\begin{array}{l}\text { Modo en que se realiza la ejercitación } \\
\text { física (esfuerzo, frecuencia e intensidad) }\end{array}$ & Ordinal & 1 & $=$ camina menos de $1 \mathrm{~h} /$ día \\
\hline & & & 2 & = camina más de $1 \mathrm{~h} /$ día \\
\hline & & & 3 & $=$ deporte/gimnasio menos \\
\hline & & & & 3 veces/semana \\
\hline & & & 4 & $\begin{array}{l}=\text { deporte/gimnasio más } \\
3 \text { veces/semana }\end{array}$ \\
\hline \multirow{2}{*}{$\begin{array}{l}\text { 15. Colesterol } \\
\text { total }\end{array}$} & Valor de colesterol sérico total & Ordinal & 1 & $=$ menor a $200 \mathrm{mg} / \mathrm{dL}$ \\
\hline & & & 2 & $=$ mayor a $200 \mathrm{mg} / \mathrm{dL}$ \\
\hline \multirow[t]{2}{*}{ 16. Colesterol LDL } & Valor de colesterol LDL sérico & Ordinal & 1 & $=$ menor a $100 \mathrm{mg} / \mathrm{dL}$ \\
\hline & & & 2 & $=$ mayor a $100 \mathrm{mg} / \mathrm{dL}$ \\
\hline \multirow[t]{2}{*}{ 17. Colesterol HDL } & Valor de colesterol HDL sérico & Ordinal & 1 & $=$ menor a $35-45 \mathrm{mg} / \mathrm{dL}$ \\
\hline & & & 2 & $=$ mayor a $35-45 \mathrm{mg} / \mathrm{dL}$ \\
\hline \multirow[t]{3}{*}{ 18. Triglicéridos } & Valor de triglicéridos séricos & Ordinal & 1 & $=$ menor a $200 \mathrm{mg} / \mathrm{dL}$ \\
\hline & & & 2 & $=$ mayor a $200 \mathrm{mg} / \mathrm{dL}$ \\
\hline & & & 3 & $=$ no aplica \\
\hline \multirow[t]{3}{*}{ 19. Glucemia } & Valor de glucemia basal sérica & Ordinal & 1 & $=$ menor a $100 \mathrm{mg} / \mathrm{dL}$ \\
\hline & & & 2 & $=$ entre 100 y $126 \mathrm{mg} / \mathrm{dL}$ \\
\hline & & & 3 & $=$ mayor a $126 \mathrm{mg} / \mathrm{dL}$ \\
\hline \multirow[t]{3}{*}{ 20. Creatinina } & Valor de creatinina sérica & Ordinal & 1 & $=$ menor a $1,3 \mathrm{mg} / \mathrm{dL}$ \\
\hline & & & 2 & $=$ entre 1,3 y $2,0 \mathrm{mg} / \mathrm{dL}$ \\
\hline & & & 3 & $=$ mayor a $2,0 \mathrm{mg} / \mathrm{dL}$ \\
\hline \multirow[t]{2}{*}{ 21. IECA } & Nombre del medicamento IECA & Nominal & 1 & $=$ enalapril \\
\hline & & & 2 & = captopril \\
\hline
\end{tabular}


Tabla 1 (continuación)

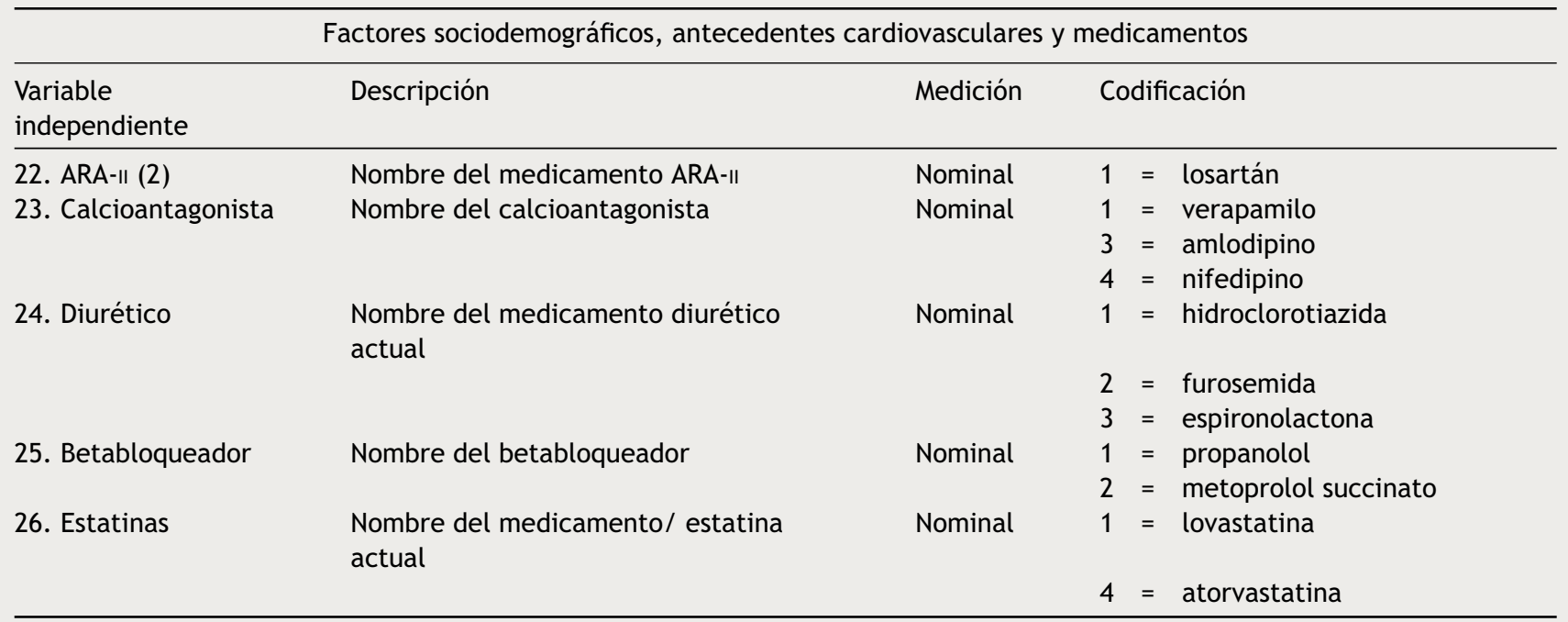

ARA-॥: antagonistas del receptor de angiotensina ॥; IECA: inhibidores de la enzima convertidora de angiotensina.

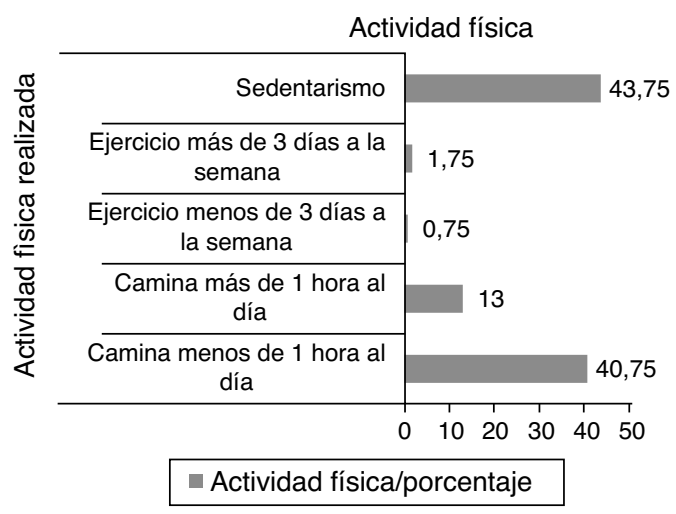

Figura 3 Distribución por actividad física.

estratificada por edad fue del $84,86 \%$ en el grupo de 50 a 60 años, del $86,72 \%$ en el de 61 a 70 años, del $81,11 \%$ en el de 71 a 80 años y del $82,22 \%$ en el grupo poblacional mayor a 80 años.

\section{Control metabólico de la población estudiada}

Dentro de los parámetros metabólicos analizados se revisó el control de colesterol total, HDL y LDL; en este último, en pacientes con diagnóstico de diabetes mellitus tipo 2, en quienes el control metabólico más estricto disminuye ostensiblemente el riesgo cardiovascular, el 1\% tenía control adecuado, tomando como cifras normales valores por debajo de $75 \mathrm{mg} / \mathrm{dL}$. Adicionalmente, se analizó el control de las cifras de triglicéridos y se observó que entre los pacientes con adecuado control, un $45,8 \%(n=183)$ eran mujeres y un $20,8 \%(n=81)$ hombres. En el $14,3 \%$ de los pacientes incluidos en el estudio no se reportaron triglicéridos séricos.

Para el análisis de las cifras de glucemia basal se establecieron tres grupos de pacientes: cifras inferiores a $100 \mathrm{mg} / \mathrm{dL}$ en ayunas, glucemia basal entre 100 y $126 \mathrm{mg} / \mathrm{dL}$ y, por último, cifras superiores a $126 \mathrm{mg} / \mathrm{dL}$. De los pacientes hipertensos analizados sin alteraciones relacionadas con los niveles de glucemia basal $(52 \%)$, un 35\% $(n=142)$ fueron mujeres y un $17 \%(n=68)$ hombres; así mismo, de los pacientes hipertensos con glucemia basal mayor a $126 \mathrm{mg} / \mathrm{dL}$ (5\%), un $3,8 \%(n=15)$ eran mujeres y el $1,2 \%(n=5)$ hombres. La glucemia basal analizada desde los diferentes grupos poblacionales incluidos, evidenció mayor proporción de pacientes con diagnóstico clínico de hiperglucemia de ayuno ${ }^{7}$ y diabetes en el grupo entre 50 - 60 años.

En la tabla 2 se muestran los resultados de los valores de colesterol total, HDL, LDL; triglicéridos, glucemia y creatinina de los pacientes incluidos en el análisis.

\section{Consumo declarado de medicamentos en la población estudiada}

El estudio analizó la prevalencia del consumo de medicamentos para el control de las cifras de presión arterial por grupos farmacológicos. Se describió la prevalencia del consumo de diuréticos, inhibidores de la enzima convertidora de angiotensina, antagonistas del receptor de angiotensina II, beta-bloqueadores, antagonistas de los canales de calcio, hipolipemiantes, antidiabéticos orales e insulinoterapia (tabla 3).

\section{Discusión}

El estudio pone de manifiesto aspectos relacionados con el riesgo cardiovascular en la población hipertensa que asiste a la consulta de atención primaria en una población rural colombiana. Se han encontrado asociaciones respecto al estilo de vida de habitantes de las ciudades; por ejemplo, menor grado de actividad física por el uso de medios de transporte, lo cual conlleva sedentarismo y aumento del peso corporal, mayor nivel de estrés relacionado con actividades cotidianas, malos hábitos dietéticos asociados con 
Tabla 2 Control metabólico en la población hipertensa

\begin{tabular}{lcrr}
\hline \multicolumn{1}{c}{ Parámetro bioquímico $(\mathrm{mg} / \mathrm{dL})$} & Frecuencia & Porcentaje $(\%)$ \\
\hline Colesterol total & $>200$ & 188 & 47 \\
& $<200$ & 212 & 53 \\
Colesterol LDL & $>100$ & 293 & 73,3 \\
Colesterol HDL & $<100$ & 107 & 26,7 \\
& $>35-45$ & 260 & 65 \\
Triglicéridos * & $<35-45$ & 140 & 35 \\
Glucemia & $>200$ & 79 & 23 \\
& $<200$ & 264 & 77 \\
Creatinina & $<100$ & 210 & 52,5 \\
& $100-126$ & 170 & 42,5 \\
& $>126$ & 20 & 5 \\
& $1,3-2$ & 362 & 90,5 \\
\end{tabular}

HDL: lipoproteínas de alta densidad; LDL: lipoproteínas de baja densidad.

* 57 pacientes sin reporte de triglicéridos.

el consumo de alimentos con altos porcentajes de grasa, hidratos de carbono y azúcares refinados, y más altos niveles de contaminación ambiental y sonora ${ }^{8}$. Cabe mencionar que se encontró mayor prevalencia de mujeres hipertensas $(69,8 \%)$, una tendencia que se refleja en gran parte de los estudios concernientes al tema; de hecho, se ha establecido que después de los 50 años de edad, la presión arterial aumenta más que en los hombres y continúa aumentando hasta los $80 \operatorname{años}^{9,10}$. De otro lado, se evidenció que la mayor parte de la población estudiada $(72,8 \%)$ tenía diagnóstico de hipertensión arterial de más de cinco años de evolución, lo que permitió correlacionarlo con la naturaleza crónica de la enfermedad.

Se encontró alta prevalencia de hipertensión arterial controlada $(84,3 \%)$, que sugiere un manejo ambulatorio adecuado y puede, a su vez, relacionarse con la prevalencia baja de comorbilidades asociadas (infarto agudo del miocardio, enfermedad cerebrovascular, insuficiencia cardíaca). Esto contrasta con las bajas tasas de hipertensión arterial controlada reportadas por estudios europeos como el PRESCAP ${ }^{11}$, el CLUE $^{12}$ y el DICOPRESS ${ }^{13}$, con cifras del 36,1, 42 y 19,4\% respectivamente. En otras palabras, el común denominador de trabajos de investigación es el control inadecuado de las cifras de presión arterial en la mayoría de los pacientes, pese a que gran porcentaje de estos se hallaba en tratamiento farmacológico. Así mismo, en la población hipertensa estudiada se encontró prevalencia de tabaquismo en un $9,3 \%$, mientras en el estudio español DICOPRESS ${ }^{13}$ fue del 22,3\%, y en el estudio con población urbana colombiana ${ }^{14}$, esta fue del $50 \%$ en el grupo de hipertensos y del $11,8 \%$, en la población general. El suceso es relevante si se considera que el tabaquismo asociado con la enfermedad cardiovascular preexistente eleva de manera exponencial el riesgo de sufrir un evento catastrófico cardiovascular en cualquier lecho vascular susceptible. Estos datos sugieren una posible efectividad de las campañas contra el tabaquismo desde la prevención secundaria, teoría que debe ser corroborada por estudios de tipo longitudinal.

De otra parte, se observó sedentarismo en un 43,5\% de la población, dato que difiere con la tasa reportada en el estudio poblacional colombiano ${ }^{14}$ en estratos socioeconómicos altos de Bogotá, donde alcanzó el 75\% y en aquellas reportadas por la Encuesta Nacional de Salud en $2007^{15}$ y la Encuesta Nacional de Situación Nutricional en Colombia en $2010^{16}$ donde supera el $70 \%$. Esto sugiere la intervención de posibles aspectos relacionados con el estilo de vida en población rural, donde gran parte de las actividades laborales se relacionan con la agricultura y la ganadería, y la mayoría de los desplazamientos se realizan caminando, dada la inexistencia de medios de transporte masivos.

Respecto al control metabólico se evidenció una prevalencia de hipercolesterolemia del 53\%, mientras que en el estudio colombiano de Ramírez et al. publicado en $2012^{17}$, esta fue del $27,1 \%$ en 147 trabajadores. Es notable la alta prevalencia total $(73,25 \%)$ y estratificada por sexo y edad para colesterol LDL elevado. En estudios europeos como el DICOPRESS la prevalencia total de colesterol LDL elevado fue del $51,1 \%$. Esta alta prevalencia de colesterol LDL no controlado en nuestro estudio podría estar relacionada con aspectos como el consumo de alimentos con elevada carga glucémica y de grasas saturadas, la inaccesibilidad (por costo elevado y oferta del mercado) a alimentos bajos en contenido calórico y sin grasas saturadas, y aspectos culturales asociados con los hábitos dietéticos de las distintas regiones del país. Se evidenció hipertrigliceridemia en el $19,4 \%$, porcentaje que puede generar baja formulación y por tanto consumo reducido de fibratos (gemfibrozilo). Se observó hiperglucemia de ayuno (glucemia basal entre $100 \mathrm{mg} / \mathrm{dL}$ y $126 \mathrm{mg} / \mathrm{dL}$ ) en el $42,5 \%$, lo que se traduce, desde el punto de vista metabólico, en riesgo elevado relacionado con evolución hacia diabetes mellitus porque indirectamente se podría tener correspondencia con síndrome metabólico y resistencia a la insulina ${ }^{18}$. Es válido resaltar que los trastornos del metabolismo de los hidratos de carbono se asocian en un $80 \%$ con la posibilidad de muerte prematura por enfermedad cardiovascular, y que los pacientes hipertensos, como aquellos incluidos en el estudio, tienen un riesgo 2,5 veces mayor de desarrollar diabetes en los próximos cinco años ${ }^{19,20}$. Aspectos como estos deben 
Tabla 3 Distribución del consumo de medicamentos por grupo

\begin{tabular}{|c|c|c|c|}
\hline & Frecuencia & Porcentaje (\%) & Porcentaje por grupos (\%) \\
\hline \multicolumn{4}{|l|}{ IECA } \\
\hline Enalapril & 138 & 34,5 & 67,6 \\
\hline Captopril & 66 & 16,5 & 32,4 \\
\hline Total & 204 & 51,0 & 100 \\
\hline \multicolumn{4}{|l|}{$A R A-\|$} \\
\hline Losartán & 103 & 25,8 & 100,0 \\
\hline \multicolumn{4}{|l|}{ Calcioantagonistas } \\
\hline Verapamilo & 100 & 25,0 & 81,3 \\
\hline Amlodipino & 19 & 4,8 & 15,5 \\
\hline Nifedipino & 4 & 1,0 & 3,2 \\
\hline Total & 123 & 30,8 & 100 \\
\hline \multicolumn{4}{|l|}{ Diuréticos } \\
\hline Hidroclorotiazida & 332 & 83,0 & 95,4 \\
\hline Furosemida & 14 & 3,5 & 4,0 \\
\hline Espironolactona & 2 & 0,5 & 0,6 \\
\hline Total & 348 & 87,0 & 100 \\
\hline \multicolumn{4}{|l|}{ Betabloqueadores } \\
\hline Propanolol & 8 & 2,0 & 14,8 \\
\hline Metoprolol succinato & 46 & 11,5 & 85,2 \\
\hline Total & 54 & 13,5 & 100 \\
\hline \multicolumn{4}{|l|}{ Manejo de DM2 } \\
\hline Metformina & 23 & 5,8 & 31,94 \\
\hline Glibenclamida & 9 & 2,3 & 12,5 \\
\hline Metformina+glibenclamida & 28 & 7,0 & 38,9 \\
\hline Insulinoterapia & 12 & 3,0 & 16,66 \\
\hline Total & 72 & 18,0 & 100 \\
\hline \multicolumn{4}{|l|}{ Manejo de dislipidemia } \\
\hline Lovastatina & 218 & 54,5 & 88,98 \\
\hline Atorvastatina & 6 & 1,5 & 2,45 \\
\hline Gemfibrozilo & 21 & 5,3 & 8,57 \\
\hline Total & 245 & 61,3 & 100 \\
\hline \multicolumn{4}{|l|}{ Otros } \\
\hline Digoxina & 4 & 1,0 & 100 \\
\hline Prazosina & 18 & 4,5 & 100 \\
\hline Clonidina & 1 & 0,3 & 100 \\
\hline ASA & 62 & 15,5 & 100 \\
\hline
\end{tabular}

ARA ॥: antagonistas del receptor de angiotensina ॥; ASA: ácido acetilsalicílico; DM2: diabetes mellitus tipo 2; IECA: inhibidores de la enzima convertidora de angiotensina.

tenerse en cuenta al momento de diseñar estrategias pertinentes a la disminución del riesgo cardiovascular, debido a que es fundamental enfocar los esfuerzos en la prevención primordial (evitar la aparición de los factores de riesgo) y controlar los factores de riesgo una vez se detectan (prevención primaria) ${ }^{21}$.

En cuanto al consumo declarado de medicamentos se encontró que el grupo de antihipertensivos de mayor consumo en la población evaluada es el conformado por los diuréticos con un $87,2 \%$, seguido por los inhibidores de la enzima convertidora de angiotensina con un $51 \%$ y de los calcioantagonistas con un $30,7 \%$. Estas cifras contrastan con el consumo declarado de medicamentos observado en el estudio realizado en la Fundación Santa Fe de Bogotá publicado en 2012 por Alvarado, en el que se encontró que los antagonistas del receptor de angiotensina ॥, con un $54,9 \%$, seguidos de calcioantagonistas, con un $29,8 \%$, fueron los de mayor consumo por la población. Los diuréticos y los inhibidores de la enzima convertidora de angiotensina fueron los de menor consumo, con un 20,7 y $18,1 \%$ respectivamente ${ }^{6}$. Esto podría evidenciar, como lo afirman Mendis et al. ${ }^{21}$, la existencia de una brecha de desigualdad relacionada con el acceso a tratamiento y barreras socioeconómicas entre los diferentes estratos y poblaciones (rurales y urbanas). En términos prácticos podría sugerirse que los hábitos de formulación de los médicos varían de acuerdo con el nivel de complejidad de la institución, la disponibilidad de recursos, el régimen de salud del paciente y la accesibilidad y el costo de los medicamentos. Vale aclarar que el presente estudio tiene algunas limitaciones en cuanto al número 
de pacientes incluidos por grupos de edad; se tomó como base el grupo de pacientes hipertensos que asisten a control del programa de enfermedades crónicas del Servicio de Medicina Interna del Hospital Felipe Suárez, y se valoraron pacientes ambulatorios, por lo cual puede subestimarse la prevalencia de descompensación metabólica y comorbilidades asociadas a hipertensión no controlada. Se pretende aportar información que sirva de base para futuros estudios descriptivos y observacionales en otras poblaciones de similares características donde puedan valorarse asociaciones entre hipertensión arterial crónica y eventos cardiovasculares.

\section{Conclusiones}

En Colombia las mayores tasas de mortalidad en población general están relacionadas con las enfermedades cardiovasculares. Es por ello que la prevención primordial y el control de los factores de riesgo cardiovascular, deben convertirse en una prioridad, en una política pública saludable del sistema de salud, que impacte a la totalidad de la población, con el fin de disminuir la prevalencia ascendente de los denominados grandes síndromes del adulto, responsables de las mayores tasas relacionadas con morbimortalidad en población general, a través de promoción de estilos de vida saludable, realización de actividad física regular y detección temprana de factores de riesgo. El conocimiento a través de estudios poblacionales sobre el comportamiento del perfil de riesgo metabólico, el consumo declarado de medicamentos y los hábitos de riesgo relacionados con el estilo de vida de los diferentes estratos socioeconómicos, permite determinar factores que los hacen proclives al desarrollo de aterosclerosis y a diferencias relacionadas con el riesgo de sufrir eventos cardiovasculares. La primera línea de acción en atención primaria en salud está encaminada al control riguroso de la presión arterial a través de una estrategia antihipertensiva adecuada basada en el riesgo global. A pesar del amplio conocimiento y de las múltiples publicaciones que abordan la hipertensión arterial y la farmacología de los medicamentos para su correcto tratamiento, estamos lejos de trasladar a la realidad dichos conceptos y aplicarlos en forma multidisciplinaria e integral a la población hipertensa, debido a aspectos como la inercia terapéutica, el incumplimiento por parte del paciente, las barreras socioeconómicas en cuanto al acceso a medicamentos de calidad, la oportunidad de asistencia a la consulta con el especialista y el ingreso a programas de prevención y rehabilitación cardiovascular.

\section{Financiación}

- Hospital Departamental Felipe Suárez, Salamina, Caldas.

- Liga contra el Cáncer. Seccional Caldas.

- Vicerrectoría de Investigaciones y Postgrados. Universidad de Caldas.

\section{Conflicto de intereses}

Los autores declaran no tener ningún conflicto de intereses.

\section{Agradecimientos}

Agradecimientos especiales a los Doctores Eugenio Matijasevic Arcila, editor de la Revista Acta Médica Colombiana; Silvio Velásquez Mejía, Darío Mejía Gutiérrez por sus aportes a la investigación; Iván Abasolo Guerrero, gerente del Hospital Departamental Felipe Suárez de Salamina, y al Semillero de Investigación de Medicina Interna MEDICAL de la Universidad de Caldas.

\section{Bibliografía}

1. Cooney MT, Dudina AL, Graham IM. Value and limitations of existing scores for the assessment of cardiovascular risk. A review for clinicians. J Am Coll Cardiol. 2009;54:1209-27.

2. Pyorala K, de Backer G, Graham I, Poole-Wilson P, Wood D. Prevention of coronary heart disease in clinical practice: recommendations of the Task Force of the European Society of Cardiology European Atherosclerosis Society European Society of Hypertension. Atherosclerosis. 1994;110: 121-61.

3. Expert Panel on Detection, Evaluation, and Treatment of High Blood Cholesterol in Adults. Executive Summary of the Third Report of the National Cholesterol Education Program (NCEP) Expert Panel on Detection, Evaluation, and Treatment of High Blood Cholesterol in Adults (Adult Treatment Panel III). JAMA. 2001;285:2486-97.

4. Yusuf S, Reddy S, Ounpuu S, Anand S. Global burden of cardiovascular diseases: part I: general considerations, the epidemiologic transition, risk factors and impact of urbanization. Circulation. 2001;104:2746-53.

5. Grundy S, Cleeman J, Bairey N, Brewer B, Clark LT, Hunninghake DB, et al. Implications of recent clinical trials for the National Cholesterol Education Program Adult Treatment Panel III Guidelines. Circulation. 2004;110:227-39.

6. Alvarado C, Jaramillo M, Matijasevic E, Rendón I, Quitian J. Estudio poblacional de factores de riesgo cardiovascular relacionados con el estilo de vida, hallazgos electrocardiográficos y medicación actual de pacientes valorados por el servicio de Cardiología. Rev Colomb Cardiol. 2012;19: $18-22$.

7. Molina D. Diabetes. Epidemiología, fisiopatología, diagnóstico, clínica, cambios terapéuticos en el estilo de vida y tratamiento farmacológico. 1. ${ }^{\mathrm{a}}$ ed. Manizales; 2012.

8. Hanes DS, Weir MR, Sowers JR. Gender considerations in hypertension pathophysiology and treatment. Am J Med. 1996;101:10-21.

9. Vasan R, Beiser A, Seshadri S, Larson M, Kannel W, D’Agostino $\mathrm{R}$, et al. Residual lifetime risk for developing hypertension in middle-aged women and men: The Framingham Heart Study. JAMA. 2002;287(8):1003-10.

10. Rodríguez GC, Artigao LM, Llisterri JL, Moreno A, Banegas JR, Lou S, et al. Control of hypertension in elderly patients receiving primary care in Spain. Rev Esp Cardiol. 2005;58(4):359-66.

11. Banegas JR, Segura J, Ruilope LM, Luque M, García-Robles R, Campo C, et al., CLUE Study Group Investigators. Blood pressure control and physician management of hypertension in hospital hypertension units in Spain. Hypertension. 2004;43:1338-44.

12. Baranera M, Campo C, Coca A, de La Figuera M, Marín R, Ruilope LM. Estratificación y grado de control del riesgo cardiovascular en la población hipertensa española. Resultados del estudio DICOPRESS. Med Clin (Barc). 2007;129(7):247-51.

13. Anchique C, Aristizábal D, Aschner P, Cano DJ, Castillo VR, Castro J, et al. Guías de prevención primaria en riesgo cardiovascular «Tópicos selectos». Rev Colomb Cardiol. 2009;16 Supl 3:71-195. 
14. Rodriguez J, Ruiz F, Peñaloza E, Eslava J, Gómez LC. Encuesta Nacional de Salud 2007. 1. a ed. Bogotá D.C: Fundación Cultural Javeriana de Artes Gráficas; 2009.

15. Fonseca Z, Heredia AP, Ocampo PR, Forero Y, Sarmiento OL, Alvarez M, et al. Encuesta Nacional de la Situación Nutricional en Colombia (ENSIN) 2010. 1. ${ }^{\mathrm{a}}$ ed. Bogotá DC: Da Vinci Editores, Instituto Colombiano de Bienestar Familiar; 2011.

16. Ramírez R, Agredo R. El sedentarismo es un factor predictor de hipertrigliceridemia. Obesidad central y sobrepeso. Rev Colomb Cardiol. 2012;19:18-22.

17. Reaven GM, Lithell H, Landsberg L. Hypertension and associated metabolic abnormalities. The role of insulin resistance and the sympathoadrenal system. N Engl J Med. 1996;334:374-81.

18. Pearson TA, Mensah GA, Alexander RW, Anderson JL, Centers for Disease Control and Prevention; American Heart Association. Markers of inflammation and cardiovascular disease: application to clinical and public health practice: a statement for healthcare professionals from the Centers for Disease Control and Prevention and the American Heart Association. Circulation. 2003;107:499-511.

19. Gress TW, Nieto FJ, Shaha E, Wofford MR, Brancati FL. Hypertension and antihypertensive therapy as risk factors for type 2 diabetes mellitus. Atherosclerosis risk in communities study. $\mathrm{N}$ Engl J Med. 2000;342:905-12.

20. Chobanian A, Bakris G, Black H, Cushman W, Green L, Izzo J $\mathrm{Jr}$, et al. Seventh report of the joint national committee on prevention, detection, evaluation, and treatment of high blood pressure. Hypertension. 2003;42:1206-52.

21. Mendis S, Abegunde D, Oladapo O, Celletti F, Nordet P. Barriers to management of cardiovascular risk in a low resource setting using hypertension as an entry point. J Hypertens. 2004;22:59-64. 